\title{
Design of a Novel Cargo-Loader for Spacecraft Container
}

\author{
Chunsheng Yang ${ }^{\mathrm{a}}$, Bile Wan ${ }^{\mathrm{b}}$, Zaicheng Wang ${ }^{\mathrm{c}}$, Yi Lu ${ }^{\mathrm{d}}$ \\ Beijing Institute of Space Environment Engineering \\ Beijing Engineering Research Center of the Intelligent Assembly Technology and Equipment for \\ Aerospace Product \\ ayangcs426@163.com, bwannsfc@163.com, cwangzaicheng@163.com, dluyu410@sohu.com
}

Keywords: spacecraft, container, cargo-loader, automatically

\begin{abstract}
To meet the requirement of loading/ unloading large-sized spacecraft containers with IL-76 aircraft, a novel cargo-loader was designed in the study. The cargo-loader including shunt mechanism and electric control system not only could moves all-around, but also be adjusted attitudes automatically. The operation efficiency and the operational risks were greatly improved owing to the automatic alignment function. Moreover, it has great potential application in other large-sized cargos during air transportation.
\end{abstract}

\section{Introduction}

Large-sized spacecraft from domestic AIT workshop are commonly transported to the launch center by air transportation. ${ }^{(1)(2)}$ For example, the communication satellite was transported by IL-76 aircraft from Beijing to Xichang launch center in 2011, as shown in Figure1. Due to the limited space, the container is too large to be hoisted by crane onboard directly. ${ }^{(3)}$ It must be taken by auxiliary facilities to elevate, move forwards, match automatically and pull into the cabin along a series of functions. ${ }^{(4)}$ The above operations above are quite time-consuming and exhausting. Therefore, it brings the risk of scratching aircraft tail and bumping container which can easily transmit extra force to inner spacecraft. ${ }^{(5)(6)}$

In order to improve loading efficiency and enhance the quality and safety of spacecraft reliability,

(7) it is essential to design an effective cargo-loader to load/ unload large-sized spacecraft container with IL-76 aircraft cabin. ${ }^{(8)}$

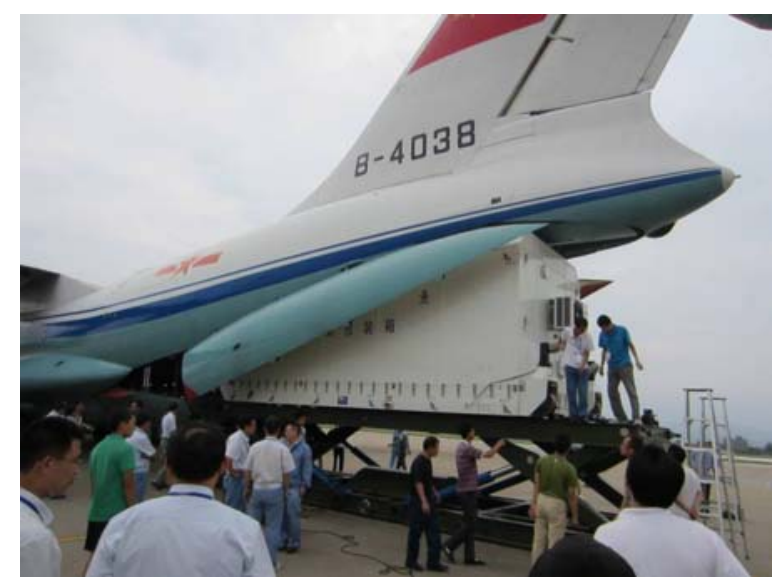

Fig.1 a communications satellite container is loading into IL-76 cabin

\section{Different modes of loading containers into aircraft}

When domestic large-sized spacecraft is transported to launch center, most of them are carried by transferring the container by air. There are overall three modes:

(1) Satellite container loading into AN124 aircraft;

(2) The container of manned-spaceship loading into IL-76 aircraft;

(3) The container of communication satellite loading into IL-76 aircraft. 


\subsection{Satellite container loading into AN124 aircraft}

Mainly, it is implemented by paving ground rail pulling by electric motor in the cabin, as shown in Figure2.

The specific process is described as follows: open up the nose of AN124 aircraft; lay rails on ground from the cabin nose towards the extension direction; put guide brackets on rails; hoist the container onto the guide brackets; pull the container into the cabin along the rail smoothly by electric motor onboard; fix the container by fixing point which locates on the bottom side and wing sides.

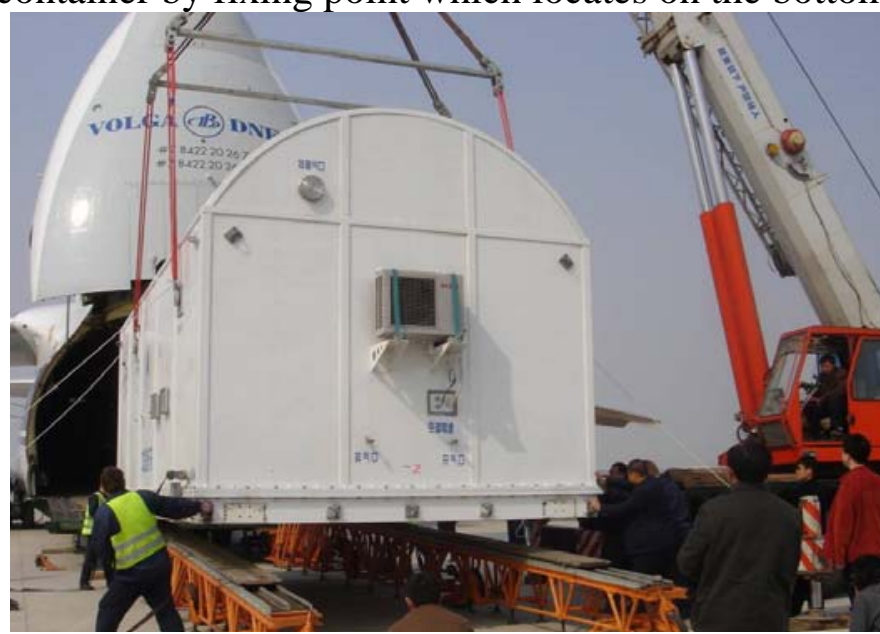

Fig.2 the container of DFH-4 platform satellite is loading into AN124 aircraft

\subsection{Manned-spaceship loading into IL-76 aircraft}

It is implemented by hoisting the container directly by the crane onboard (Figure3).

The specific process is described as follows: open up the gate of bellyhold; turn tail of the aircraft to the lowest point; transportation trolley approaches to the tail of aircraft; hoist the container by crane of the aircraft and move it smoothly into the cabin; fix the container by fixing points which locate on the bottom side and wing sides of container. This mode is suitable for those small and light containers.

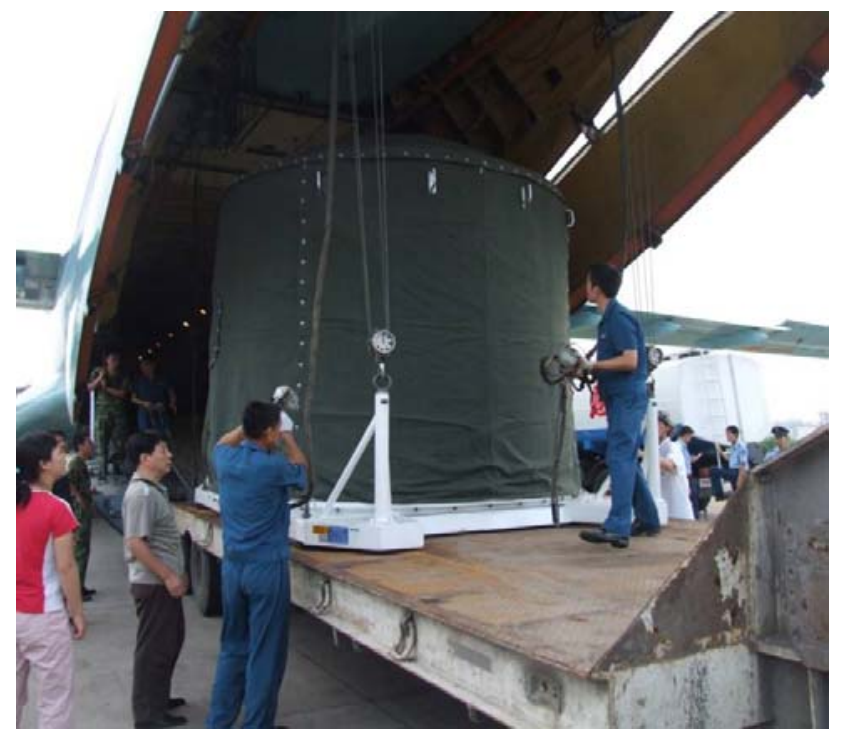

Fig.3 manned-spaceship is loading into IL-76 aircraft

\subsection{Satellite loading into IL-76 aircraft}

It is implemented by both electric cargo-loader and traction motor in cabin. Combining the above mechanisms, the container should move forwards and upwards alternately till into the cabin.

The specific process is described as follows: open up the gate of bellyhold; adjust the aircraft attitudes to keep cargo deck horizontally; mark auxiliary lines on the ground; arrange the elevator platform and low vehicle along the lines marked strictly; hoist the container onto low vehicle; operate the motor onboard to pull the container on the elevator platform; ascend the container by elevator platform till the bottom of container and deck of cabin is the same in height; align the elevator 
platform with rail onboard; pull the container into the cabin smoothly. In order to avoid the IL-76 aircraft space limit (Figure 4), the container should go forwards and upwards with great care.

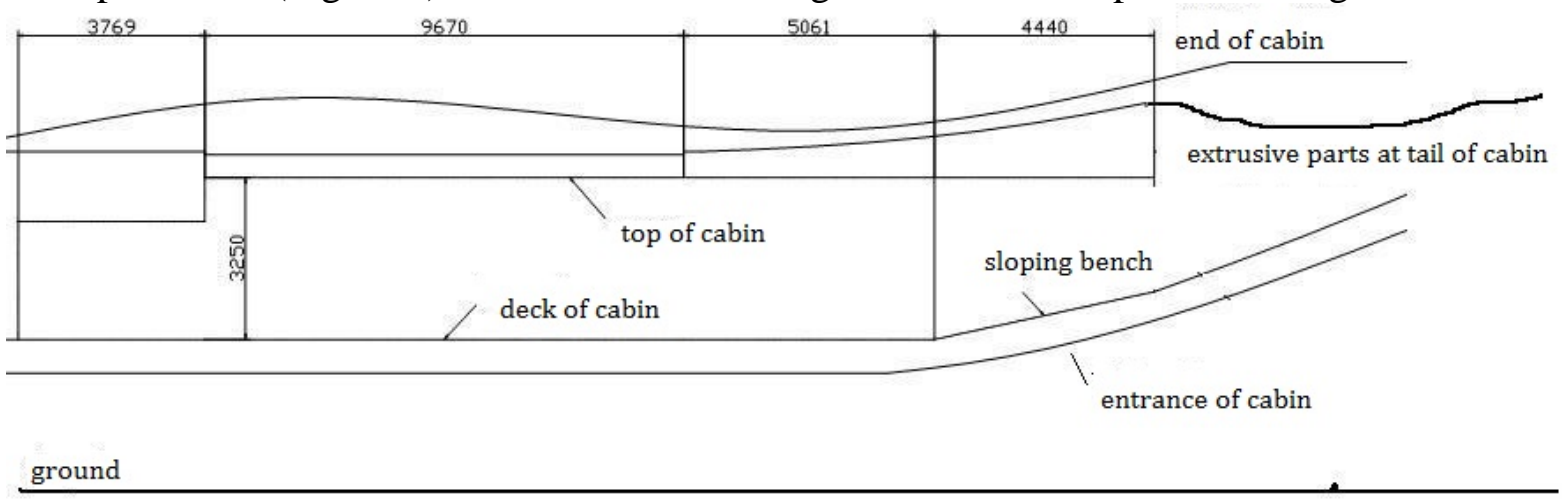

Fig.4 IL-76 aircraft tail boundary limits

\section{Cargo-loader design}

\subsection{Overall scheme of the cargo-loader}

To conveniently load the communication satellite container into IL-76 aircraft with limited space and power supplied, we designed a novel cargo-loader in the study, as illustrated in Figure5.

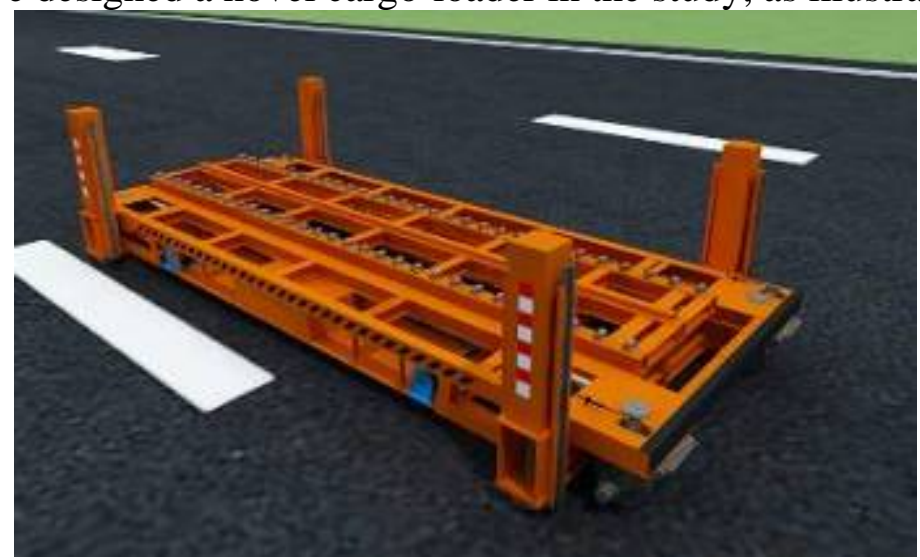

Fig.5 general view of the new cargo-loader designed

This cargo-loader is featured with parallel mechanism, and equipped with main frame platform, hydraulic rams, electric control system, steering wheels, automatic alignment system, etc. The main frame platform carries container, which can be further ascended, adjusted, and moved.

\subsection{Main body structure of the cargo-loader}

The cargo-loader is composed of the cart platform (marked 1 in the picture below), upper layer working platform (marked 2), hydraulic control system (marked 3), pitching cylinders (marked 4), steering and fixing wheels (marked 5), the main elevating cylinders (marked 6), electrical control cabinet (marked 7), winch system (marked 8), steering wheels (marked 9), anti-collision rubber pad (marked 10), a pair of cross roll-control cylinders (marked 11), and related accessories, as depicted in Figure6. 


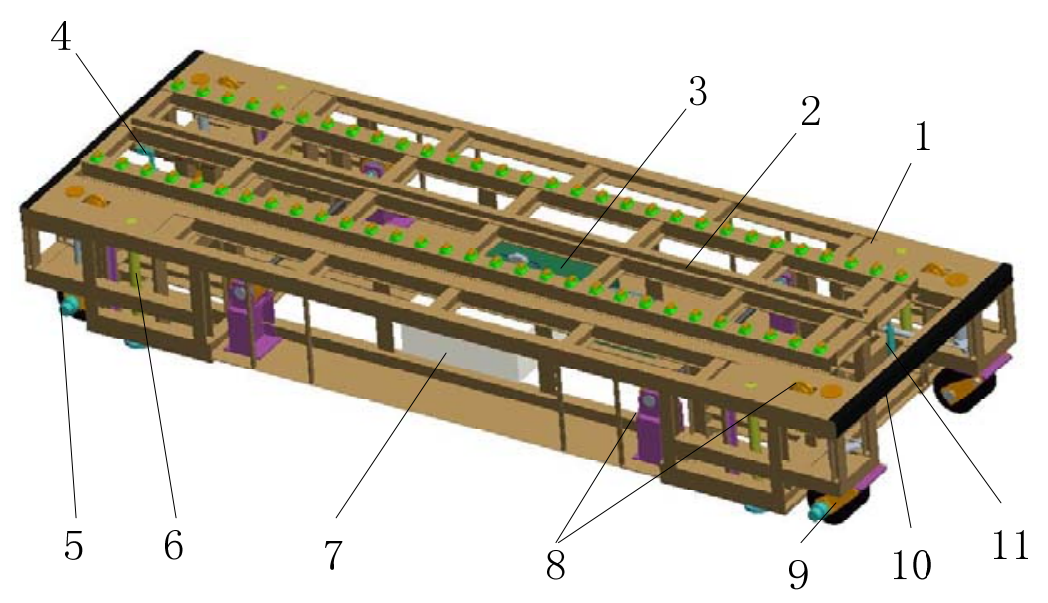

(a) Mechanical components of the cargo-loader

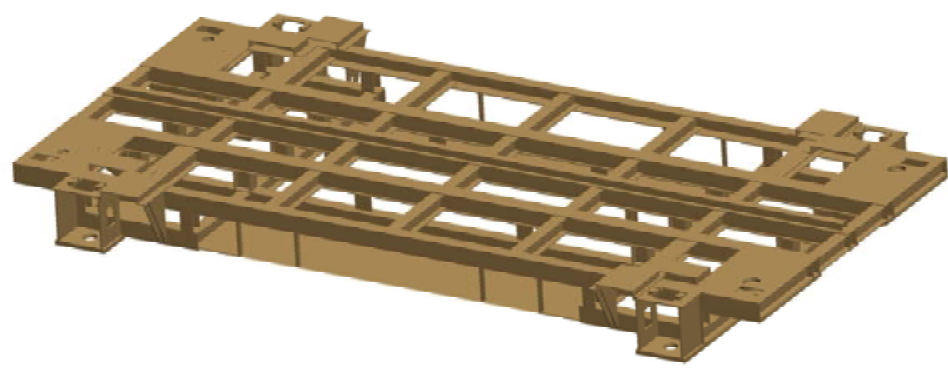

(b) the cart platform

Fig. 6 mechanical components of the cargo-loader

The frame of cart is welded together. Upper layer working platform above the cart can pitch was rolled and moved by connecting the pitching cylinder and crossed roll-control cylinders with cart platform. When pitching cylinder stretched, the front side of platform will be ascended, so the whole cart can pitch. When crossing roll-control cylinders work synergically, the upper layer working platform can roll. Rolling and pitching is independent from each other.

On the top of layer platform, there are two rails which contact container directly by pulley rolling. Hydraulic control system and electric control cabinet is arranged at the bottom of the cart.

The cargo-loader carries the container loading into cabin or unloading to the transport trolley. There are track rails both on the cart platform and on the bottom of container. All of the above rails can be mated with aircraft well. The cart should be designed with enough support stiffness to keep rails supporting steadily.

The outline dimension of the cargo-loader is shown as below:

(1) Total length: $9200 \mathrm{~mm}$;

(2)Total width: 3420mm;

(3) Elevating range: $0 \sim 1500 \mathrm{~mm}$.

\subsection{Electric control system}

The electric control system mainly functions in controlling the power supply, mating the cargo-loader with transportation vehicle, and aligning cargo-loader with cabin.

The electric control system is consisted of two various cabinets (power cabinet and program logic control (PLC) cabinet) and an operating platform. The power cabinet is used to .supply the electric power. The PLC cabinet is constituted by a master station and a slave station. The PLC cabinet controls the displacement sensor, absolute value encoder, incremental encoder, a variety of proximity switches in signal detection, and the servo valve and proportional valve. The module with high speed and high precision is used as the signal acquisition and control output. Then the corresponding speed and control precision can be improved. The operating platform is movable and manipulated by buttons, indicating lamps, operating levers, and a wired operation handle. 


\subsection{Main function}

This cargo-loader can work either supported by four wheels or by four cylinders. By transferring between these two modes aforementioned, the cargo-loader can achieve the function of all-round self-moving, lifting and adjusting.

Four wheels supporting the cargo-loader is shown as in Figure7.

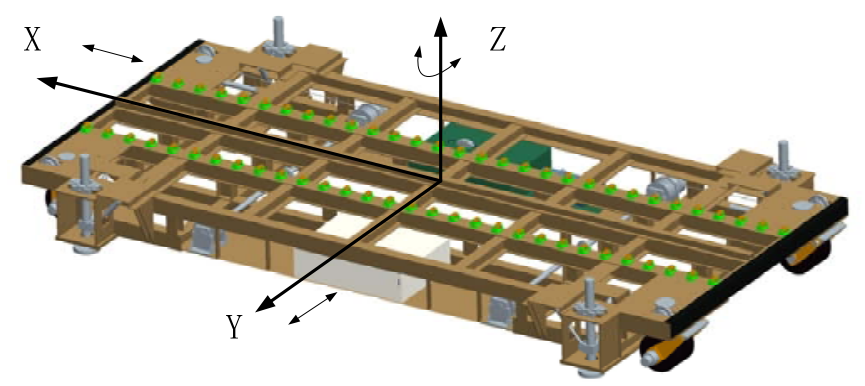

Fig.7 four wheels supporting the platform when moving

Working with this mode, the cargo-loader can achieve the following four kinds of movement:

(1) Moving along X directions: four wheels constitute a surface which is in parallel with the X direction, and work synchronously to make the cargo-loader move;

(2) Moving along Y directions: four wheels rotate 90 degrees inwards. Then surface is in parallel with the Y direction, and the four wheels work synchronously to make the cargo-loader move;

(3) Rotating in situ: the central line of every wheel is vertical to the turning axis, so four wheels rotate inwards.. The four wheels work synchronously to make the cargo-loader rotate as expected;

(4) Moving towards any horizontal directions: the cargo-loader moves along X direction and Y direction alternately. It can move to any directions by change the adjacent paces.

In the process of the above functions, the steering range of four wheels is $0-90$ degree.

The cargo-loader supported by four cylinders supporting is depicted in Figure 8.

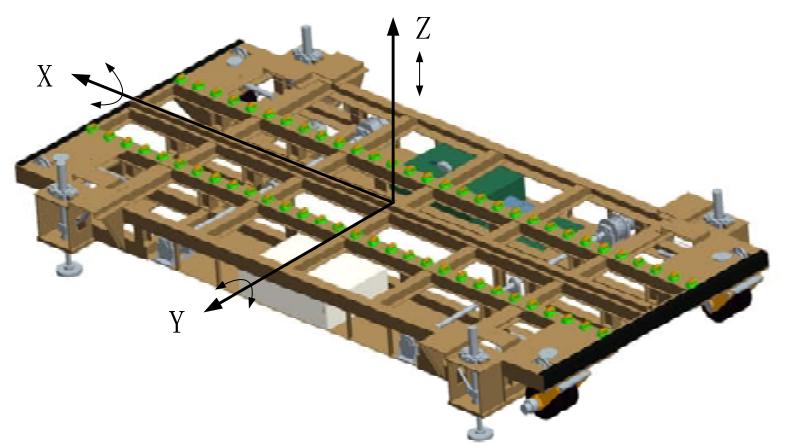

Fig.8 four cylinders supporting the platform when adjusting

In this mode, the cargo-loader can achieve the following three types of movement:

(1) Moving along $\mathrm{Z}$ directions: Based on four main cylinders extend synchronously, the landing legs will approach to the ground gradually. After three landing legs of them contact the ground, the cargo-loader will be controlled according to force feedback, the cargo-loader will transfer to distance feedback until all of four landing legs touch the ground.

(2) Rotating around the Y axis: main cylinders on one side (forwards or backwards) are locked, and cylinders on the opposite side rise. The transverse sides of cart ascend correspondingly at the same time. Then vertical cylinders should be unlocked, working following the cart. Thus the cargo-loader can pitch.

(3) Rotating around the $X$ axis: main cylinders on one side (left or right) should be locked, and the opposite side rise. Meanwhile the same side of cart will ascend. Then cross roll-control cylinders should be unlocked, working following the cart. So the cargo-loader can roll.

Combining rolling around both $\mathrm{X}$ axis and $\mathrm{Y}$ axis, the cargo-loader can adjust the attitudes by itself. 


\subsection{Layout of mechanism}

The layout of mechanism is comprised of four wheels, four cylinders, two groups of winch, hydraulic station, and electric control appliances together, as shown in Figure 9. Among them, four cylinders are the key components.

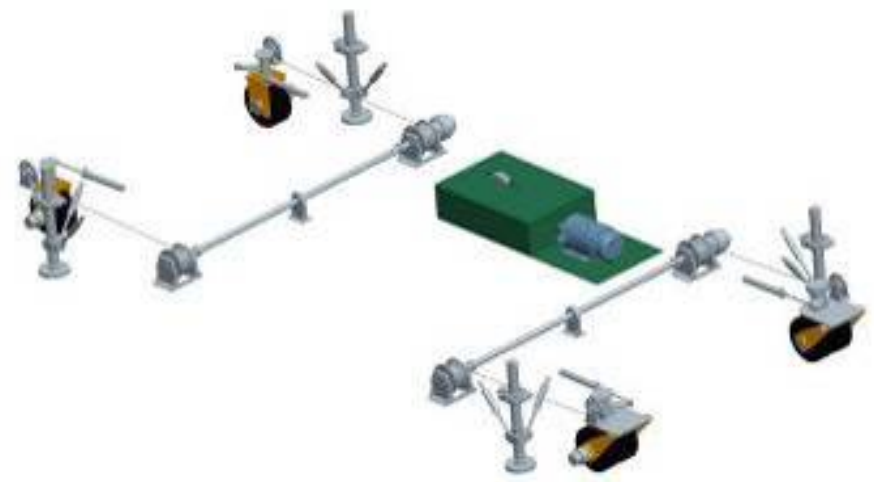

Fig.9 layout of mechanism

According to the requirements of working conditions, the structure includes main elevating cylinders (marked 1 in the picture below), forks with two degrees of freedom (marked 2), vertical sway cylinders (marked 3) and transverse sway cylinders (marked 4), as shown in Figure10.

Elevating cylinders are connected with the cart by forks with two degrees of freedom. The direction position of the cart is controlled by two sway cylinders. The vertical sway cylinder controls pitching, while the transverse sway cylinder master rolling.

Both the guide beam and the guide way can move along main elevating cylinders, so the main cylinder can take load off, as shown in the Figure 10.

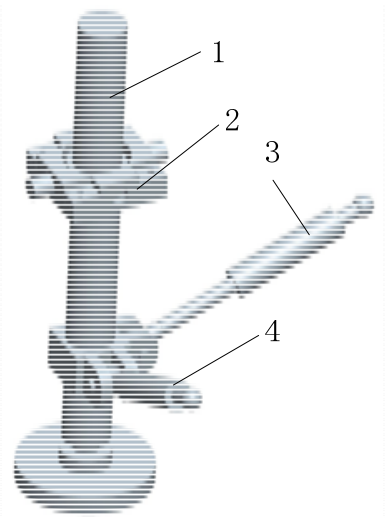

(a) main cylinder

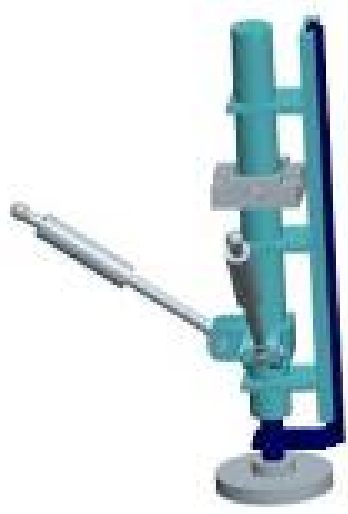

(b)load-off structure

Fig.10 system of main cylinder

Considering factual rugged ground conditions, the cargo-loader is designed with two overturning height-nonadjustable wheels at the front and two overturning height-adjustable wheels at the back resulting that four wheels undertake similar force. The height of wheels should be adjusted by cylinders. The oil-current of the whole cylinder system is isolated from the others. But among the inner four cylinders, the oil-current is connected directly. So the two wheels can be adjusted automatically according to the height of the ground. Meanwhile, they can afford holding force for each other.

\subsection{Automatic alignment function}

This new cargo-loader is equipped with laser rang finders, infrared sensors, ultrasonic sensors. By both operators viewing and the above sensors measuring before automatically adjust itself, the cargo-loader can mate with the cabin well. ${ }^{(3)}$

The cargo-loader carrying container approaches to the cabin; then cargo-loader rotates along vertical axis till the guide rail is parallel with the rail onboard and the plane of the container bottom is in the same height with cabin deck; Infrared sensors transmit infrared light to the bellyhold, then we can observe the infrared light on cabin to mate cargo-loader with cabin preliminarily, as shown in the Figure 11. Then, laser rang finders work to adjust the cargo-loader precisely. The laser range finders 
locate on both sides of the front. According to the measured distance differences, the cargo-loader adjusts its attitudes. the cargo-loader is elevated to the same height with cabin by ultrasonic sensors detecting, as shown in the Figure 12. Based on the above measurements, the container can move into the cabin correctly.

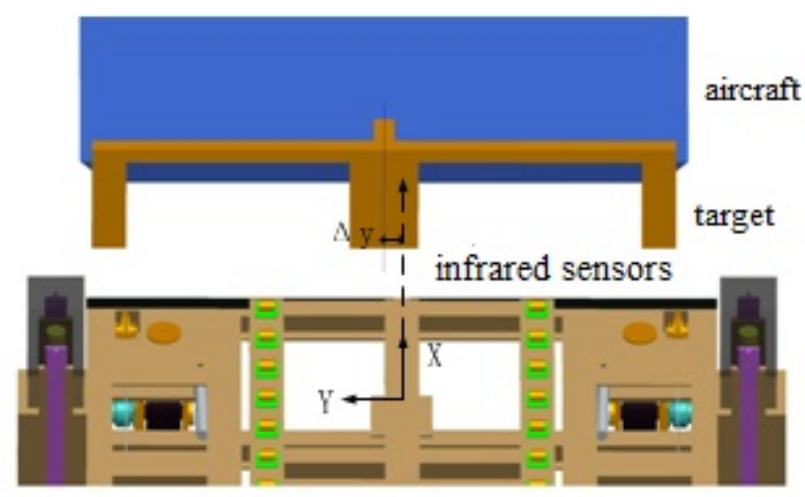

Fig.11 mating by infrared light preliminarily

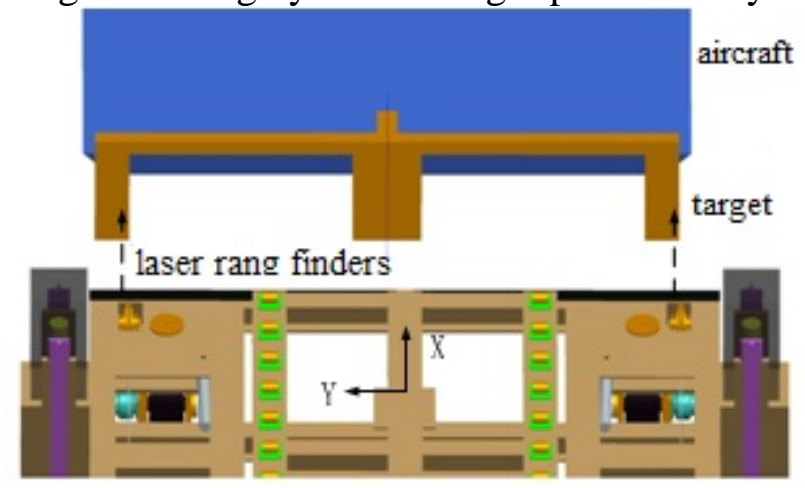

Fig.12 mating by laser range finder precisely

\section{Security analysis of cargo-loader}

\subsection{Safety and reliability design}

The cargo-loader is powered by hydraulic drives with large load carrying capacity, compact structure, fast speed, the cargo-loaded proposed meets the requirements of stability and safety during loading and unloading. Additionally, it can meet the requirements of loading and unloading by the hydraulic motor movement synchronization control technology. The minimum speed of motor is more than $0.5 \mathrm{rpm}$ and the minimum offset can achieve $5-8 \mathrm{~mm}$. Therefore, it can meet the requirements of mating alignment accuracy.

In the design process, mechanical design safety factor is 5 , winch cable safety factor is 5 , the cylinder design safety factor is more than 5, and redundancies of motor driving force is more than 3 .

There are sufficient protection measurements involved in this cargo-loader, for example:

(1) The main cylinders can be locked with double channels;

(2) There are two groups of auxiliary cylinders (4 for per group), and the cargo-loader can work safely when any of the two cylinders run well;

(3) Each cylinder is provided with a lateral unloading structure;

(4) Movement of container is controlled by winch at two directions;

(5) The interference during loading can be solved by 3 methods: manual observation, sensors detection and the position adjustment;

(6) It is forbidden to make the four wheels and four main cylinders work synchronically, which are interlocking;

(7) Pitching, rolling, moving is independent. When any action is in the implementation, the other movements are locked; 
(8) In emergency, the cargo-loader can be stopped and all the components will be locked immediately.

\subsection{Feasibility analysis}

The configuration is optimized, the structure is simple, and it can meet all the requirements of container movement. Capacity of cargo-loader is large and speed is low due to equipped with hydraulic power, meeting the requirements of the stability and safety during loading and unloading. Hydraulic motor is controlled synchronously so that the rotating speed and displacement can meet the requirements of alignment accuracy.

\subsection{Advantages and characteristics}

The whole process operation is easy to manipulate, convenient to control. And it needs less devices and less crew members. Efficiency is obviously improved: process steps are reduced; the position and attitude adjustment time is greatly shortened; the movement repetition rate is reduced, so it takes less time during loading and unloading.

The technology level is significantly upgraded: pitching, rolling is independent from each other; the above movement can also work synchronously at any time if needed.

Performance is excellent: automatic detection by various sensors, manually preliminarily setting, cargo-loader adjusting can be combined so the whole process is in good accordance with the practical condition. Besides, total cost is low, technical difficulty is moderate, system complexity is normal, maintenance is convenient.

\section{Summary}

This paper presents an effective cargo-loader, which can provide technical reference for other large-sized containers of spacecraft during loading and unloading.

The new cargo-loader can overcome disadvantages of loading mode mostly adopted presently. Meanwhile, it is more convenient, and efficient with low-risk. It can also solve the difficulties of other large-sized containers loading into aircraft.

\section{References}

[1]. Emma Jones. New Approaches for Autonomous Logistics Aircraft and Ground Systems. Autonomous Systems, 2007 Institution of Engineering and Technology Conference. 23-23 Nov. 2007

[2]. James Michael Snead. Configurable Air Transport. AIAA 2005-814

[3]. Xiao Gang. The development of containers for air transport of spacecraft and an evaluation. Spacecraft Environment Engineering. Vol. 27(2010) No.6, p. 795-799.

[4]. M. E. Franke, Gurler Ari. Use of Cargo Aircraft for Launching Precision-guided Munitions. AIAA 2004-1250

[5]. AMSS-Aircraft Maintenance Support Services Ltd. Jane’s Airports, Equipment and Services. 20-May-2011

[6]. Myles A. Rohrlick. Load-by-Wire Advanced Cargo Handling System. AIAA and SAE, 1997 World Aviation Congress, Anaheim, CA, Oct. 13-16, 1997, 8 p.

[7]. Air Force Procurement of 60K Tunner Cargo Loader Contractor Logistics Support (D-2006-059). March, 2006

[8]. James Michael Snead. Configurable Air Transport. AIAA 2005-814 